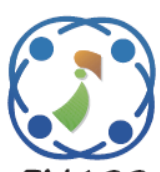

\title{
No reference Image Quality Measure for Hazy Images
}

\author{
Ahmed Rafid Hashim ${ }^{1}$ \\ Hazim G. Daway ${ }^{2 *}$ \\ Hana H. kareem ${ }^{3}$ \\ ${ }^{1}$ Department of Computer Science, College of Education for Pure Sciences-Ibn Al-Haitham, \\ University of Baghdad, Baghdad, Iraq \\ ${ }^{2}$ Department of physics, College of Science, Mustansiriyah University, Baghdad, Iraq \\ ${ }^{3}$ Department of physics, College of Education, Mustansiriyah University, Baghdad, Iraq \\ * Corresponding author's Email: hazimdo@uomustansiriyah.edu.iq
}

\begin{abstract}
Haze causes the degradation of image quality. Thus, the quality of the haze must be estimated. In this paper, we introduce a new method for measuring the quality of haze images using a no-reference scale depending on color saturation. We calculate the probability for a saturation component. This work also includes a subjective study for measuring image quality using human perception. The proposed method is compared with other methods as, entropy, Naturalness Image Quality Evaluator (NIQE), Haze Distribution Map based Haze Assessment (HDMHA), and no reference image quality assessment by using Transmission Component Estimation (TCE). This done by calculating the correlation coefficient between non-reference measures and subjective measure, the results show that the proposed method has a high correlation coefficient values for Pearson correlation coefficient (0.8923), Kendall (0.7170), and Spearman correlation coefficient (0.8960). The image database used in this work consists of 70 hazy images captured by using a special device, design to capture haze image. The experiment on haze database is consistent with the subjective experiment.
\end{abstract}

Keywords: Color saturation, Correlation coefficient, Hazy image database, HSV color model, No-reference image quality, Subjective experiment.

\section{Introduction}

The atmosphere contains small types of suspended particles called aerosols [1]. Aerosols are fine liquid or solid particles suspended in the air. Haze, dust, fog, smoke, and drizzle are examples of these particles. Haze is a famous type of aerosol that causes problems in aerial imaging. Therefore, studies have been conducted to remove haze and then improve the quality of degraded images. In addition, a quality scale is an important indicator of the degrees of degradation and improvement. Image Quality Assessment (IQA) is well known in the scope of image processing. In [1], the IQA is classified into two main groups, namely, fidelity and intelligibility of image. Fidelity is described as the deviation grade between evaluated and standard images.

Intelligibility is able to supply information for human or computer. Image quality can be an assessment by quantitatively measuring the combined two groups. The IQA is classified into two methods, namely, subjective and objective methods. The subjective method uses human's subjective sense to estimate image quality.

This method simulates the perception technique of a people, a visual system to estimate it. The objective method estimates image quality through a machine. This method has three categories as follows [2]: Full-Reference IQA (FR-IQA), ReduceReference IQA (RR-IQA), and No-Reference IQA (NR-IQA). FR-IQA estimates the quality of a degraded image by comparing it with the reference image. The full reference method is based on Structural Similarity Index Measurement (SSIM) [3] and Universal Quality Index (UQI) [4]. RR-IQA uses finite features from the original image rather than a complete image to estimate the quality of the degraded image. RR-IQA uses limited features from a reference image rather than a complete image to 
estimate the quality of the degraded image. NR-IQA provides image quality without any reference image or its features. NR-IQA can be divided into the following two classes [5]: The first class includes the algorithms developed for specific types of distortion, such blur [6-9], jpeg [10, 11], jpeg2000 compression $[12,13]$, noise[14], and others [15-18]. The second class includes the non- distortion-specific algorithms; for example, Moorthy and Bovik [19] presented a method on the basis of a two-step framework called blind image quality index for NR-IQA using natural scene statistics. Previous knowledge of the degrading process is unnecessary once trained. Mittal et al. [20] established NIQE, an image quality metric in a spatial domain, on the basis of a natural scene statistic model, the drawback of this method is not working properly in high density.

All the aforementioned techniques used nonreference quality measures used to measure the degree of distortion. However, it is not used to measure hazy distortions in color images, which has an important application in the field of aerial photos. However, some of the following studies have suggested non-reference quality metrics to measure the quality of Hazy images as: Xiaoxi Pan et al. [21] presented a no-reference assessment using the range channel of images; a haze distribution map was extracted from hazy images; moreover, the HDMHA was used, although this algorithm is compatible with in remote sensing but is failed in the haze. Wei Shen, Shuman Hao, Jiansheng Qian, and Leida Li [22] developed a new NR quality metric for de-hazed images by measuring entropy, contrast, and luminance, this method good works at low and medium haze density but is not always work properly at high density haze. Hana Hasan et al. [23] presented a new NR quality metric for dehaze images depending on wavelet transform and transmission component by calculating the histogram of the (HL) wavelet component for transmission component, this algorithm correctly works at low and medium haze density but is not work properly at high density haze. The proposed method was compared with other methods such as entropy, HDMHW, TCE by calculating the correlation coefficient, results show that the proposed method has a high correlation coefficient values.

In the present work, we propose a new method for measuring no-reference image quality and conducting subjective experiments on the haze image database, the proposed method success in the measure the quality in all degrees of degradation. This paper also includes the following sections: sec. 2 explain image database, sec.3 explain subjective experiments, sec. 4 explain Study hazy images depending on color spaces, sec.5 explain the proposed method in detail, sec.6 explain correlation coefficient, sec.7 explain the experimental results, and sec. 8 explain the conclusion.

\section{Image database}

The famous model that used in computer vision to depict the haze image formation is [24]:

$$
I(x)=J(x) t(x)+A(1-t(x))
$$

where $I(x)$ represent the observed intensity at $x$ position, $J$ represents the scene radiance, A represents the atmospheric light, and $t$ represents the transmission medium. In this paper, a system has been designed to measure the haze level (dust is used to make haze), that is, the distorted image. Because there is no database for hazy distorted images, and with distortion, ratios graduated from little to high. These images were generated by means of the staging design to capture these images. Fig. 1 illustrates the system, which contains a firm glass box, HeNe laser, fan, camera, camera stand, and lux meter. When the fan on the dust is raised in the glass box, the lux meter measures the laser intensity. The dust remains at the bottom of the box after a short period. During this period, the images are captured using the camera. The function of the lux meter is to read the intensity of the laser light. The intensity decreases with the increase in dust intensity, whereas it increases with the decrease in dust intensity. A total of 91 images were captured and divided into seven groups. The hazy images in each group ranging from thick haze images to little haze images see Fig. 2, the captured images are not of equal size the first group is (1274x904), the second group is $(1274 \times 745)$, the third group is (958x960), the fourth group is $(1078 \times 1040)$, the fifth group is $(458 \times 388)$, the sixth group and seven groups are $(640 \times 480)$, these images are in jpeg format. Database images can be downloaded through a link [25].

\section{Subjective experiments}

The experiment on the subjective user is consistent with the recommendation of [26]. In Experiment 35, college students comprise males and females aged between 22 and 25 years, all students have a normal vision. The students do not have specific knowledge related to IQA. Thus, their opinions are completely based on their own prior knowledge and preference. The students are asked to select an image with one of five grades, which are explained through five groups as follows: "bad," "poor," "fair," "good," and "excellent" (regarding 


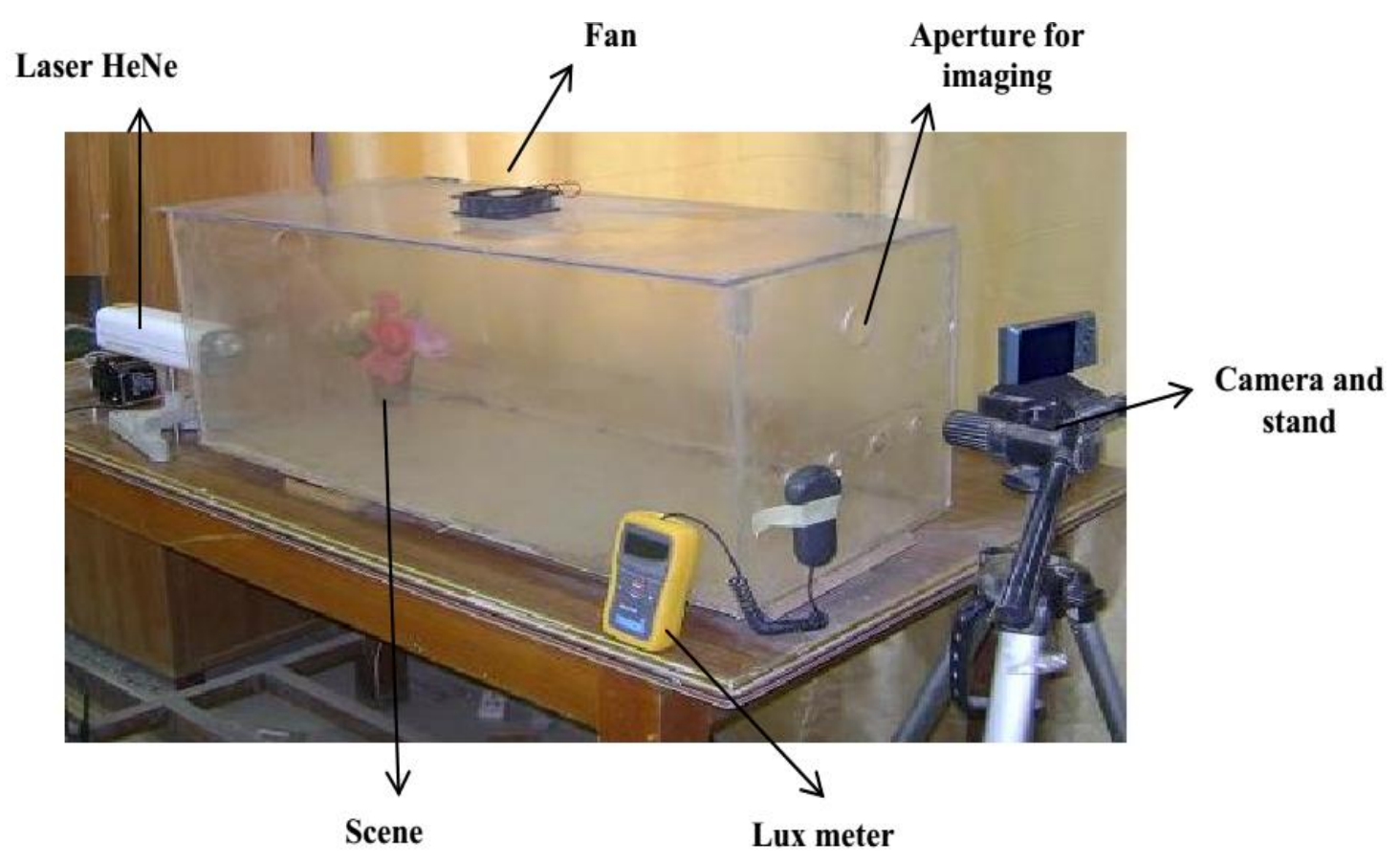

Figure. 1 System used to capture hazy images

scores from 1 to 5), these images used were displayed using 16 inch screen with steady lighting and the distance between screen and viewers one meter. According to [26], the time required to complete one experiment must not exceed $30 \mathrm{~min}$, and each image requires 15 seconds, including 10 second for observation and 5 seconds for scoring. The database has 70 images, and the file is in jpg format. The final value of the Mean Opinion Score (MOS) for each image has been acquired by averaging all quality evaluations for a given image. Subjective experiment results can be downloaded through a link [25].

\section{Study hazy images depending on color spaces}

Color Space plays an important role in color image processing. In these systems, color is defined as a point in 3D space and quantified. These spaces are designed as a function of in red, green and blue components, color spaces can be divided as follows basic color space (RGB) Color spaces based on the Human Visual System or Television Color Spaces (as YIQ, YCbCr and YUV), Perceptual Color Spaces which can be divide as (HSV), (HLS) and HIS, and Perceptual non Uniform Color spaces as (LUV) and (LAB). In this research, the effect of the hazy (dust) on the image will be studied based on the color spaces to propose a no-reference image quality assessment. We will try to answer the question which color space can be used mathematically in the scale? A brief summary of the space will be taken as we will study the distribution of the histogram of the spaces (RGB,
YCbCr, LAB and HSV), as explained in Fig. 3 which acts the relationship between the component and the probability of images (from a high level of distortion to dust to a low level of distortion) for each color space. In RGB color space we notice difficulty in finding a clear relationship between the level of distortion and the change in distribution for each component due to the high correlation in the compounds. Also in the $\mathrm{YCbCr}$ and LAB spaces, no significant changes were observed in the distributions. In the HSV space, it is possible to observe that there is a good relationship between the degree of distortions and the range of the saturation component, which can be used in the no-reference scale. Fig. 4 explain the relationship between saturation and haze for the same image as the saturation decreases as the haze increases and increases saturation as less saturation. This behavior is reflected in the values of the non-reference scale where $Q$ value decreases due to the increase of distortion.

\section{Proposed method (no reference objective)}

In this paper, we use a new method for noreference quality of a hazy image. This method consists of the following steps:

a. Convert an image from RGB to hue, saturation, and value (HSV) color space because the latter matches human's visual sensing better than the former and other color models. HSV application is for human interface. Fig. 5 depicts the 


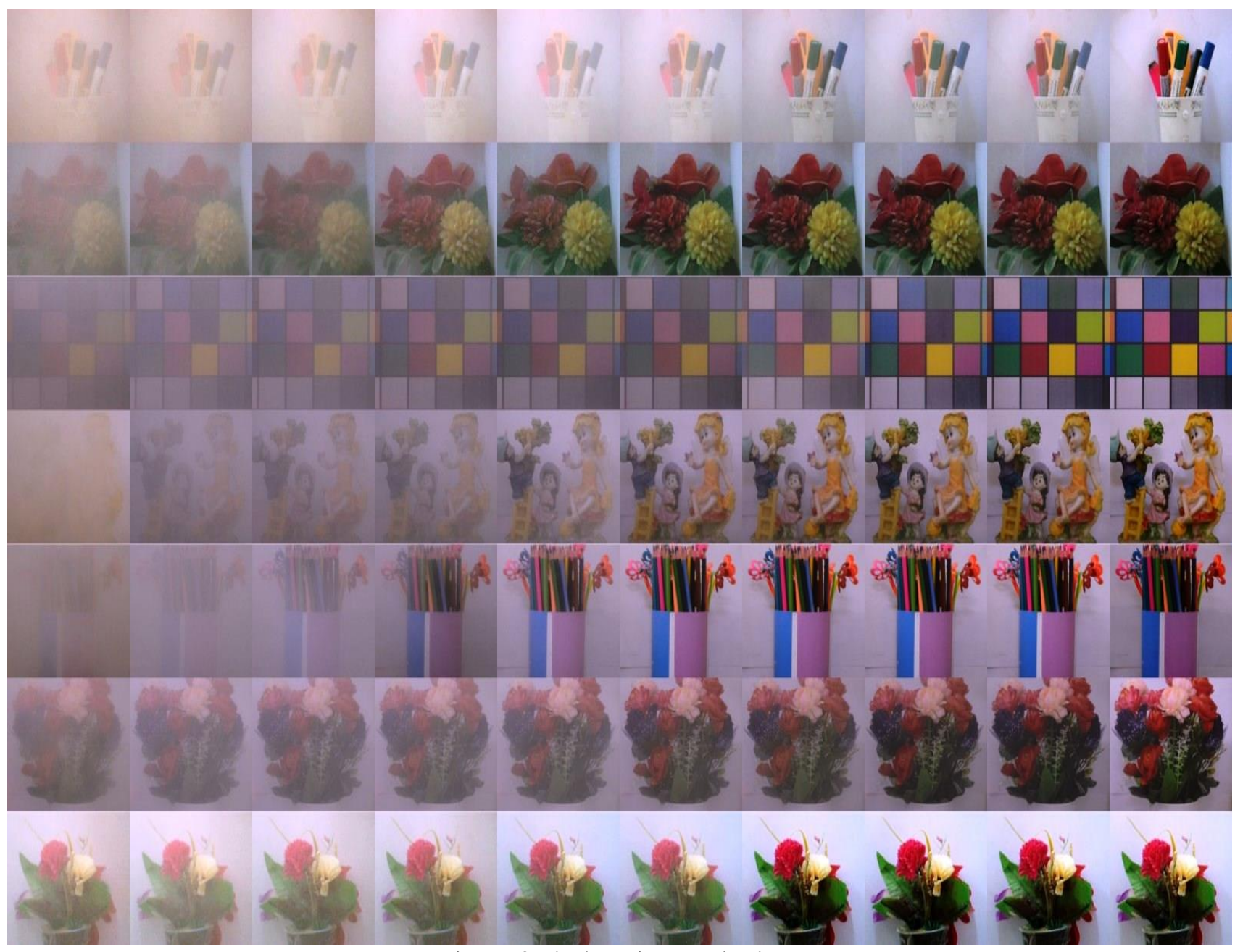

Figure. 2 The hazy images database

HSV color model. HSV explains a color model in terms of three components [27]. Hue represents the type of color (example red, green, blue, magenta, cyan, and yellow).

That values from $0^{\circ}$ to $360^{\circ}$. Considering the saturation point to the intensity of specific hues, saturation is also called "purity" of colors or the amount of gray in colors. Saturation ranges from $0 \%$ to $100 \%$ or 0 to 1 , where 0 represent gray, and 1 represent a primary color. Value refers to the colors brightness. It values from $0 \%$ to $100 \%$ or from 0 to 1 , where 0 refers to black. When value increases, colors appear bright. Convert RGB to HSV using a conversion formula, in which values of $R, G$, and $B$ divided by 255 to change the range from $0-255$ to $0-1$ [27]:

$$
\begin{aligned}
& \mathrm{R}^{\prime}=\mathrm{R} / 255 \\
& \mathrm{G}^{\prime}=\mathrm{G} / 255 \\
& \mathrm{~B}^{\prime}=\mathrm{B} / 255
\end{aligned}
$$

and

$$
\begin{aligned}
C \max = & \max \left(\mathrm{R}^{\prime}, \mathrm{G}^{\prime}, \mathrm{B}^{\prime}\right) \\
\mathrm{Cmin}= & \min \left(\mathrm{R}^{\prime}, \mathrm{G}^{\prime}, \mathrm{B}^{\prime}\right) \\
& \Delta=\mathrm{Cmax}-\mathrm{Cmin}
\end{aligned}
$$

The Hue calculation [27]:

$$
H=\left\{\begin{array}{cc}
0^{\circ} & \Delta=0 \\
60^{\circ} *\left(\frac{C^{\prime}-B^{\prime}}{\Delta} \bmod 6\right) & , C \max =R^{\prime} \\
60^{\circ} *\left(\frac{B^{\prime}-R^{\prime}}{\Delta}+2\right) & , C \max =C^{\prime} \\
60^{\circ} *\left(\frac{R^{\prime}-C^{\prime}}{\Delta}+4\right) & , C \max =B^{\prime}
\end{array}\right.
$$

The Saturation calculation [27]:

$$
S=\left\{\begin{array}{cc}
0 & , C \max =0 \\
\frac{\Delta}{C \max } & , C \max \neq 0
\end{array}\right.
$$



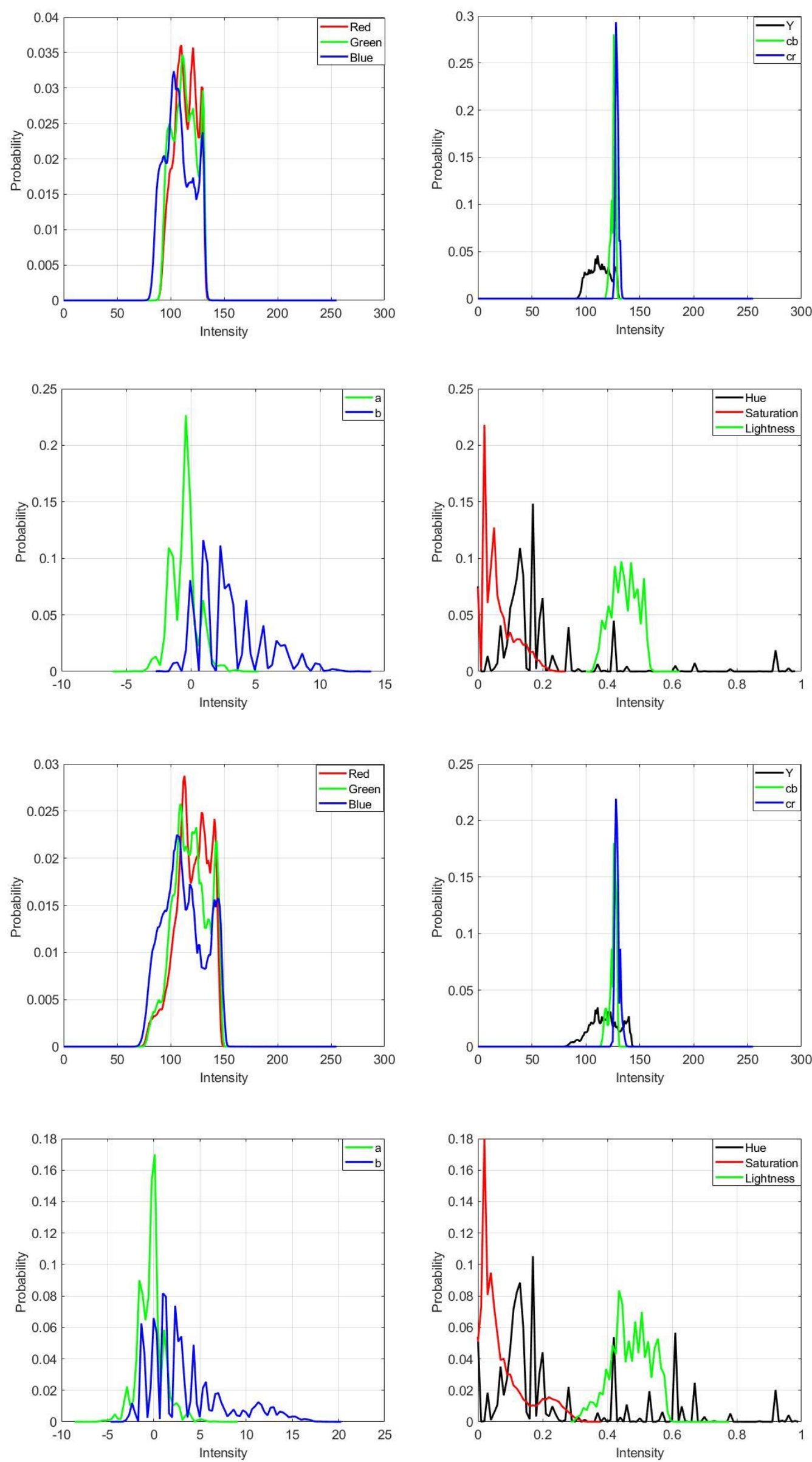

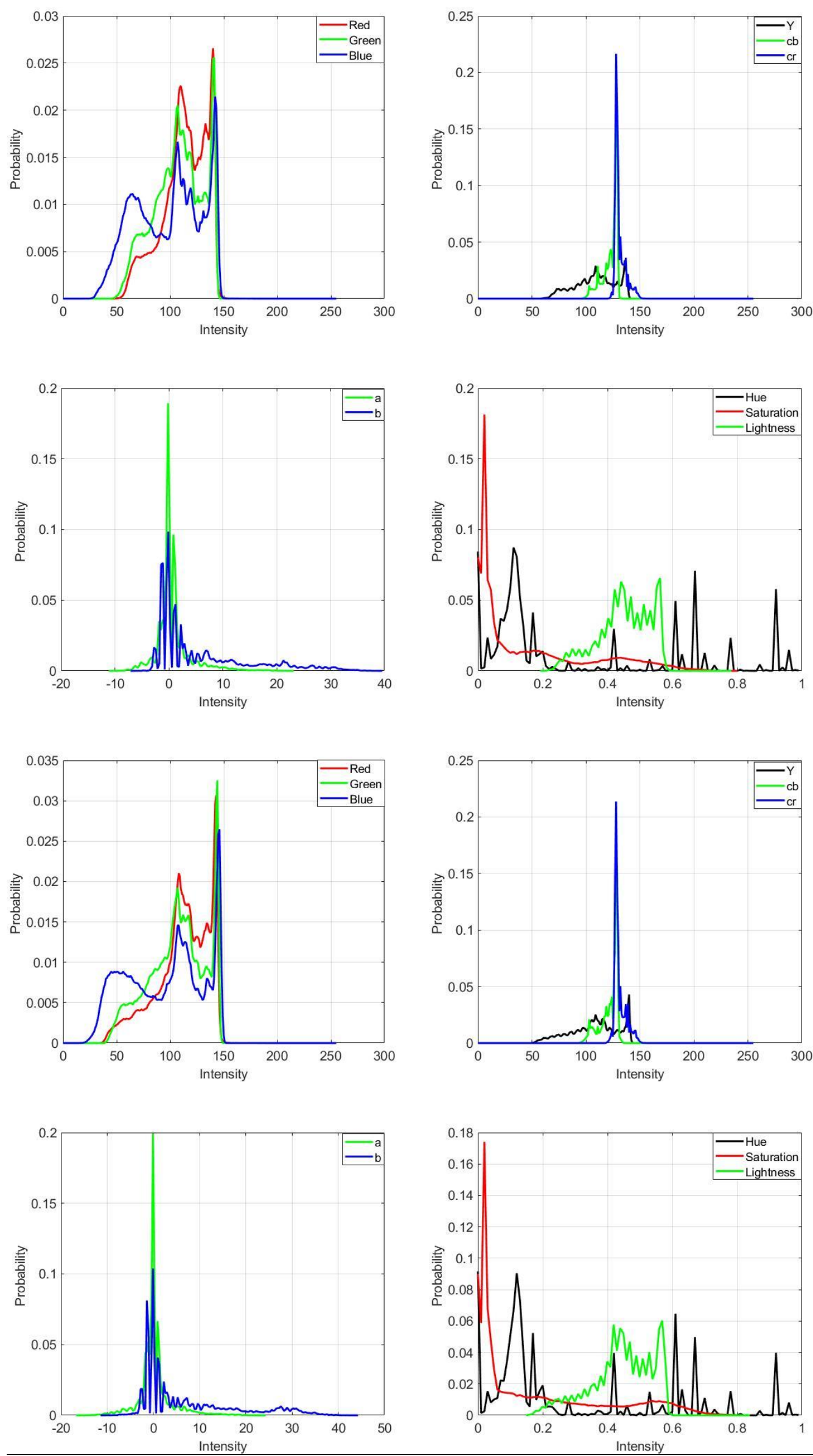

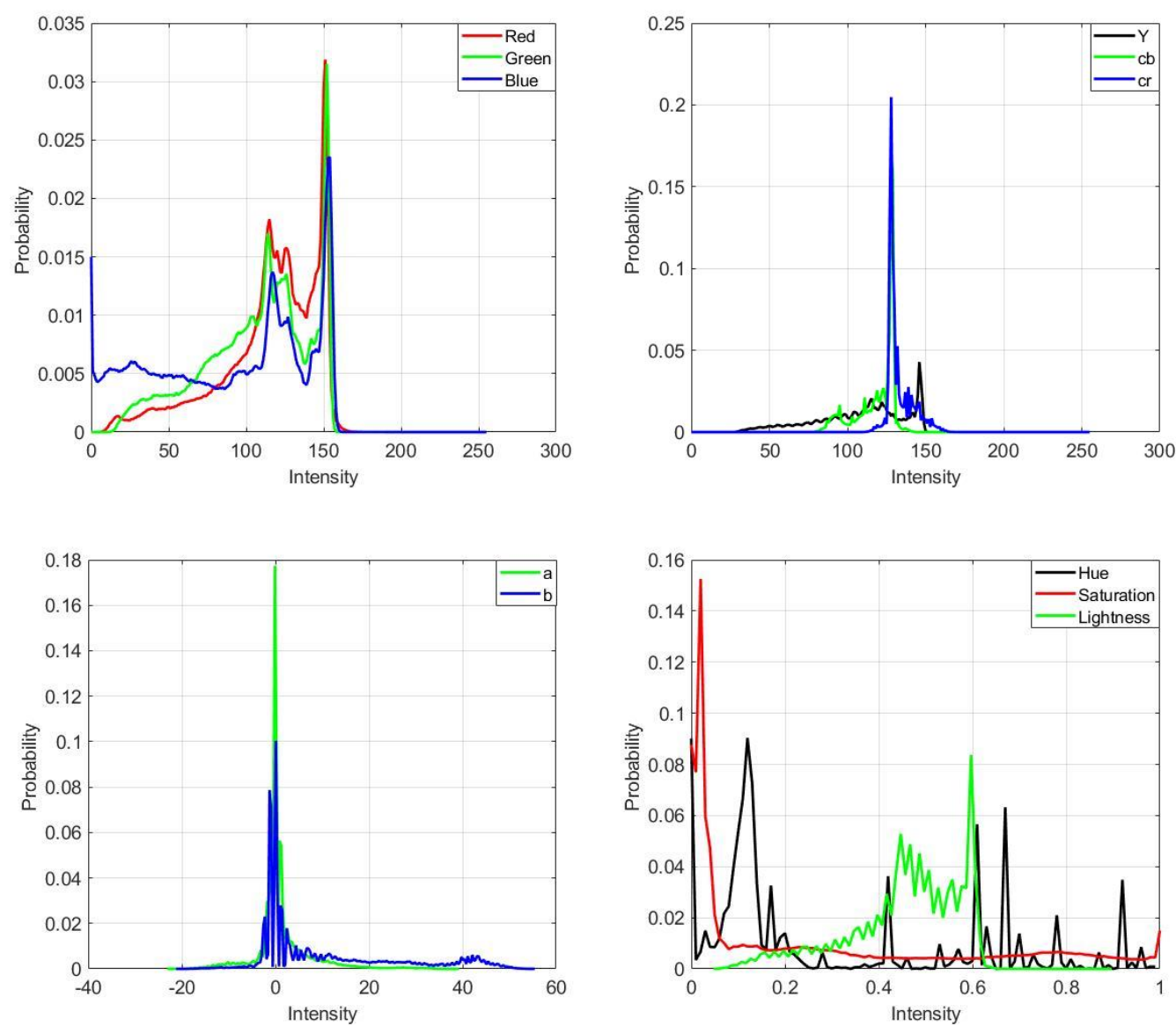

Figure. 3 Distribution of histograms of hazy images (at high to low distortion levels) using color spaces RGB, YCbCr, LAB and HSV for each component, except the lightness component (L) in the L

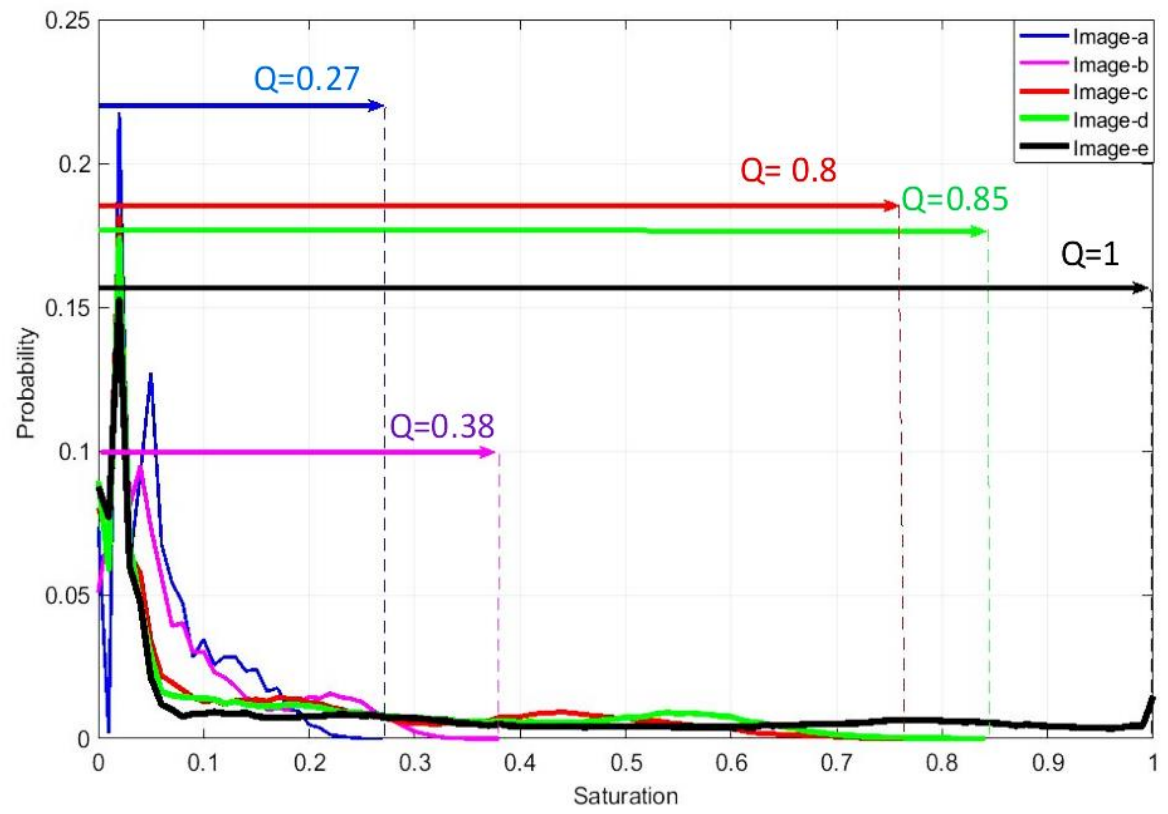

Figure. 4 No-reference quality assessment for different images

Value calculation [27]:

$$
V=C \max
$$

b. Extract saturation component from HSV color.by using Eq. (9). 


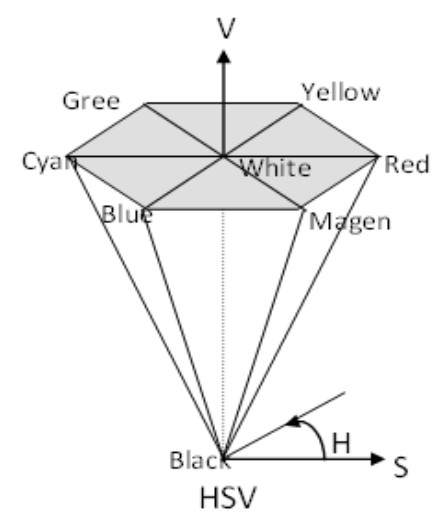

Figure. 5 HSV color model

c. Calculate the frequency for saturation component $(S)$, with two accurate digits after the comma, where $S$ normalizes $(0-1)$. Where $d$ refers to the two digits after the comma.

$$
S t=\operatorname{round}(d \times S) / d
$$

Calculate the probability of saturation. Fig. 6 demonstrates a set of hazy images with the calculated probability and their corresponding 3D-HSV model.

These images progress from thick haze to minimal haze. An increase in haze affects the distribution of saturation probability, thereby diminishing the saturation component. The distribution of saturation component is proportional to haze, and the distribution of saturation toward the least area in the histogram is drawn, thereby reducing the quality value. Moreover, in the 3D-HSV model, the size of the solid region becomes small and increases with the decrease in the value of haze.

$$
\operatorname{Pr}=\text { frequency }(S t) / \text { size of image }
$$

d. Estimate the quality of the proposed method

$$
\text { Quality }=\max (S t)-\min (S t)
$$

\section{Correlation coefficient}

Correlation estimates the strength of the relationship between two variables. The present work uses the following correlation coefficient.

\subsection{Pearson correlation coefficient (PCC)}

PCC is applied to obtain the dependency between two variables. A PCC range is between " -1 " and " $+1 "$. The value near to "+1" indicates that the two variables have a positive correlation [2]. By contrast, the value near to "-1" indicates that the two variables have a negative correlation. Low value or zero value indicates that the two variables are not correlated. The PPC $(p)$ between two variables " $\mathrm{x}$ " and " $\mathrm{y}$ " with standard deviations $\sigma \mathrm{x}$ and $\sigma \mathrm{y}$, correspondingly, is presented as follows [2]:

$$
p=\frac{\text { convariance }(x, y)}{\sigma x \sigma y}
$$

\subsection{Spearman correlation coefficient (SCC)}

SCC supplies the relation between two variables. It ranges from " -1 " to " +1 " with the same work as that of the PCC. The SCC is calculated as follows [2]:

$$
p=1-\frac{6 * \sum r^{2}}{n\left(n^{2}-1\right)}
$$

Where $(r)$ represents the difference in ranks of two variable $(x)$ and $(y)$.

\subsection{Kendall rank correlation coefficient (Kendall's tau coefficient)}

Similar to the SCC, Kendall rank correlation coefficient estimates the relationship between two variables. Kendall correlation is calculated as follows [28]:

$$
\begin{aligned}
& k=\frac{\sum_{i=0}^{n} \sum_{j=1}^{n} \operatorname{sgn}(x i-x j) \operatorname{sgn}(y i-y j)}{n(n-1)} \\
& \operatorname{sgn}(x i-x j)=\left[\begin{array}{c}
1 \text { if }(x i-x j)>0 \\
0 \text { if }(x i-x j)=0 \\
-1 \text { if }(x i-x j)<0
\end{array}\right] \\
& \operatorname{sgn}(y i-y j)=\left[\begin{array}{c}
1 \text { if }(y i-y j)>0 \\
0 \text { if }(y i-y j)=0 \\
-1 \text { if }(y i-y j)<0
\end{array}\right]
\end{aligned}
$$

This coefficient measures the discrepancy between the number of concordant and discordant pairs. Any two pairs of ranks $(x i, y i)$ and $(x j, y j)$ are concordant when $x i<x j$ and $y i<y j, x i>x j$ and $y i<y j$, or $(x j-y j)(y i-y j)>0$. Furthermore, any two pairs of ranks $(x j, y j)$ and $(x j, y j)$ are discordant when $x i<x j$ and $y i>y j, x i>x j$ and $y i<y j$, or $(x j-y j)(y j-y j)<0$. Like the two previous correlation coefficients, Kendall rank correlation coefficient values from -1 to +1 , with the absolute value of $\mathrm{k}$ sign the strength of the relationship between the two variables [28]. Also, Kendall rank correlation coefficient can be 1 for a wider value of scenarios than the SCC. 


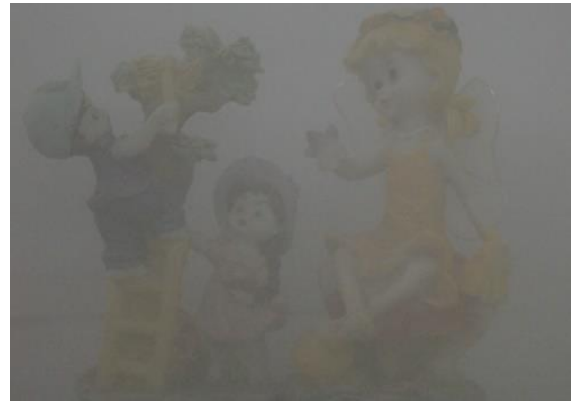

(a)

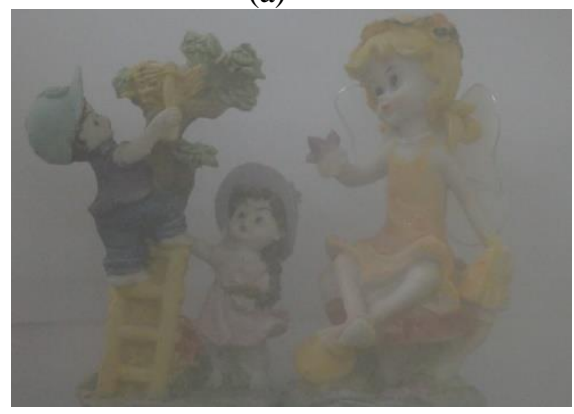

(d)

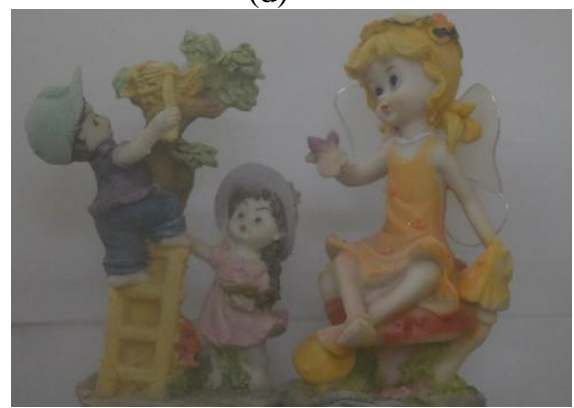

(g)

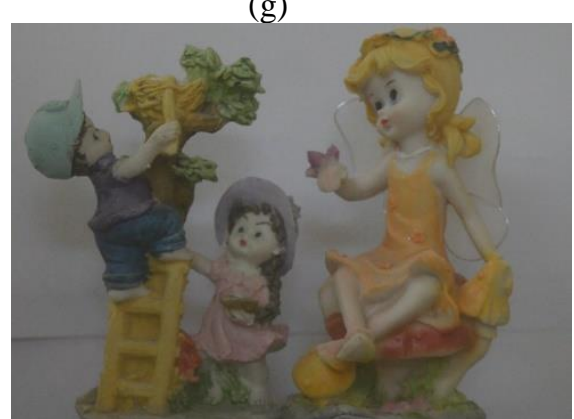

(j)

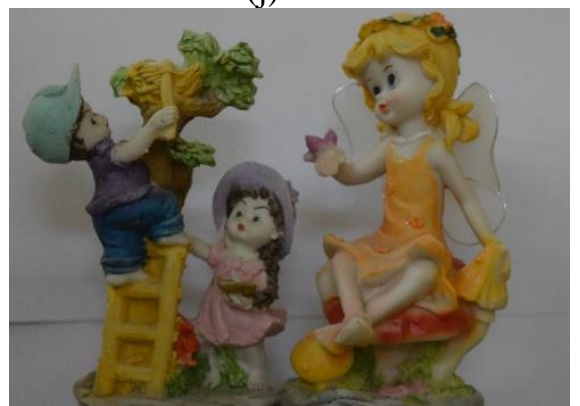

(m)

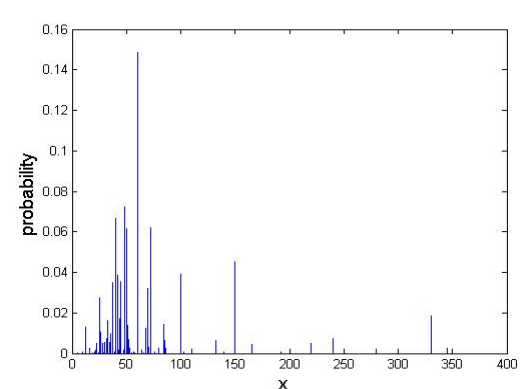

(b)

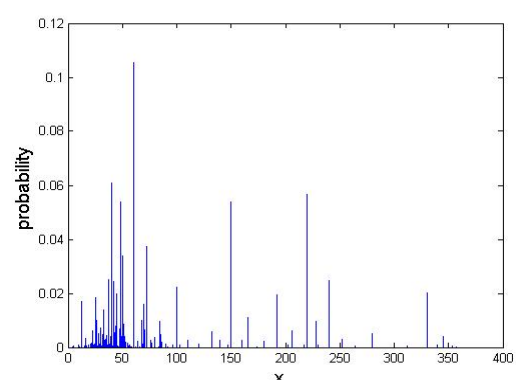

(e)

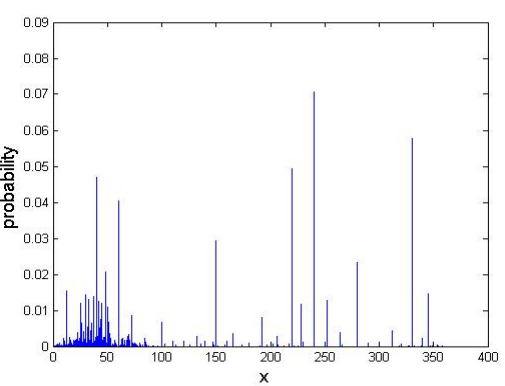

(h)

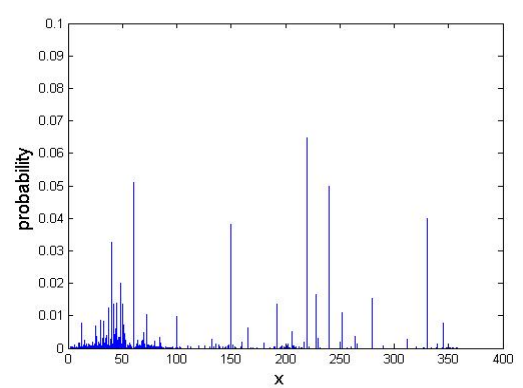

(k)

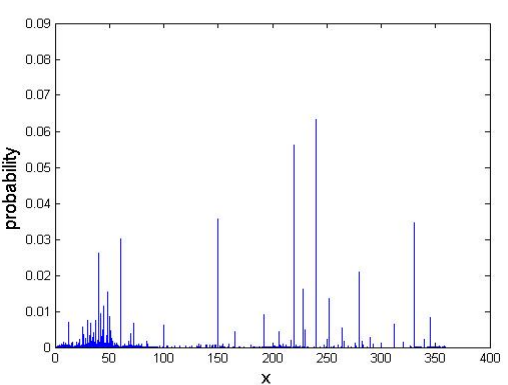

(n)

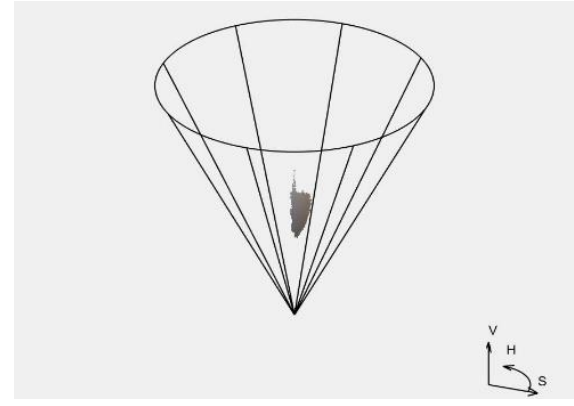

(c)

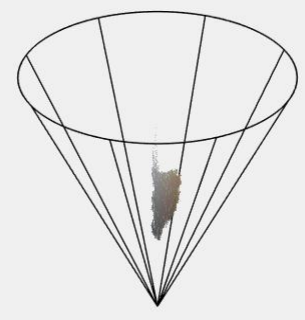

(f)

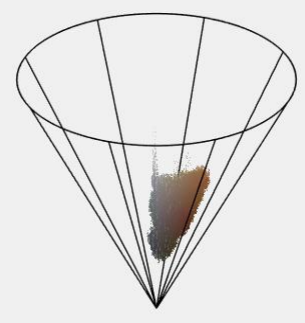

(i)

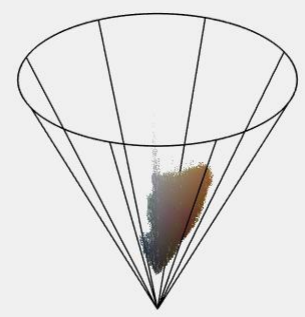

(1)

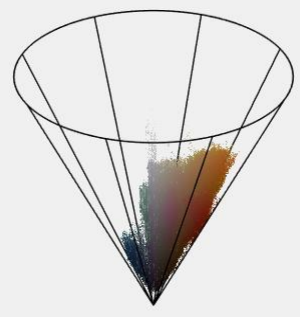

(o)

Figure. 6 Five hazy images with the histogram for a saturation component that corresponds to the HSV model: (a) image (1), (b) histogram of saturation for image (1), (c) 3D HSV model for Image (1), (d) Image (2), (e) Histogram of saturation for image (2), (f) 3D HSV model for Image (2), (g) Image (3), (h) Histogram of saturation for image (3), (i) 3D HSV model for Image (3), (j) Image (4), (k) Histogram of saturation for image (4), (1) 3D HSV model for Image (4), (m) Image (5), (n) Histogram of saturation for image (5), and (o) 3D HSV model for Image (5) 
Table 1. Correlation coefficient values between the proposed and the other methods

\begin{tabular}{llll}
\hline objective methods & PCC & kendall & SCC \\
\hline Entropy & 0.6942 & 0.5131 & 0.6902 \\
NIQE & 0.4943 & 0.4209 & 0.5452 \\
HDMHA & 0.3016 & 0.2630 & 0.3761 \\
TCE & 0.4114 & 0.2879 & 0.4211 \\
proposed method & $\mathbf{0 . 8 9 2 3}$ & $\mathbf{0 . 7 1 7 0}$ & $\mathbf{0 . 8 9 6 0}$
\end{tabular}
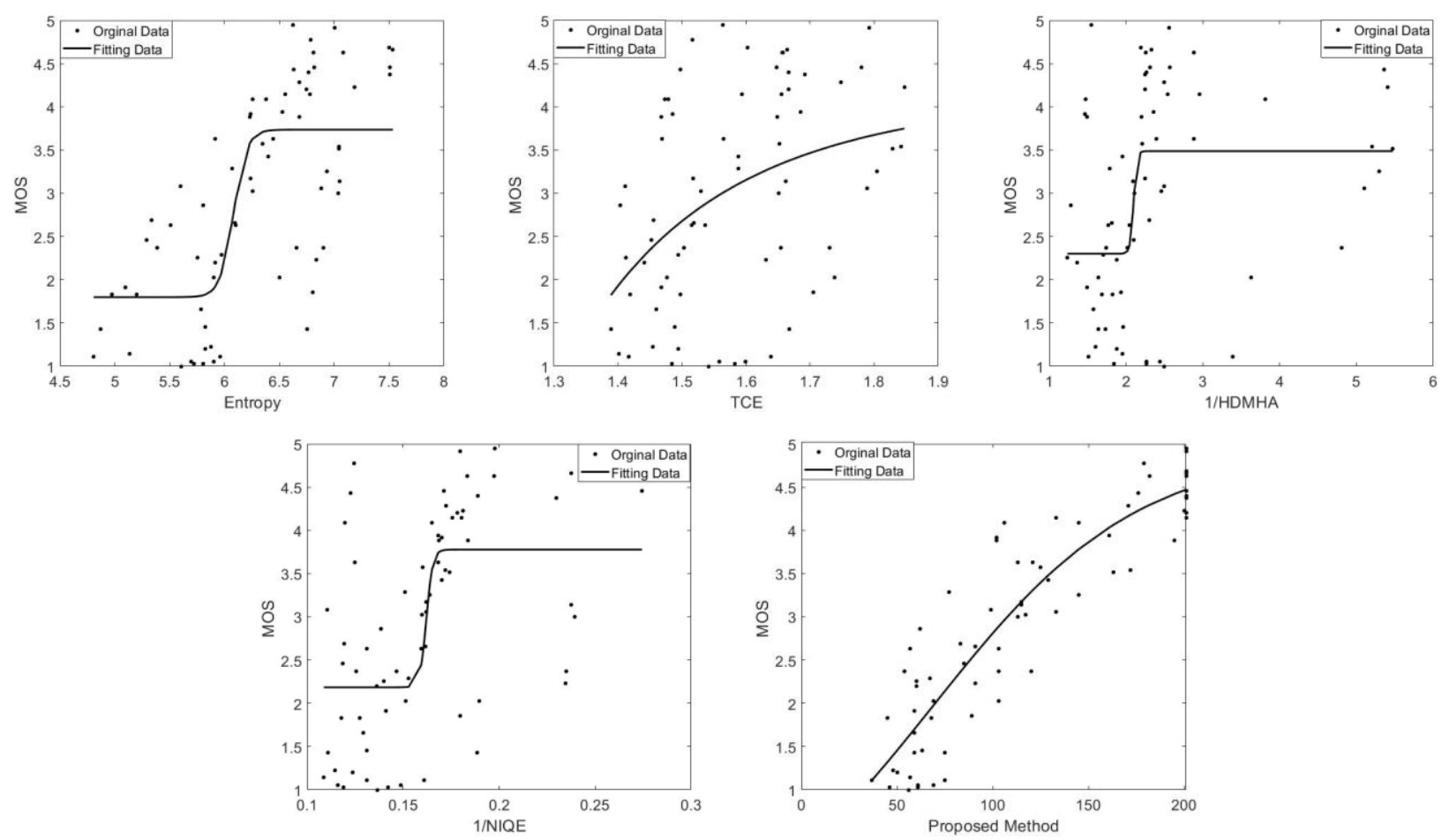

Figure. 7 Scatter plots and fitted curves of the subjective (MOS) versus the IQA methods on the haze database

\section{Experimental result}

This part explains the comparison of the presented method with three other methods, namely, entropy [22], NIQE [20], and HDMHA [21], TCE [23]. All algorithms are performed by using MATLAB version (R2013a) on a computer device which properties a processor with a $2.4 \mathrm{GHz}$ Core i5 and $4 \mathrm{G}$ RAM.

The three correlation coefficients, namely, PCC, SCC, and Kendall's tau coefficient, are used to check the performances of these methods between objective and subjective scores. The image database used in this work consists of hazy images (70 images) captured by using a special device, design to capture haze image as explain in sec.2. Fig. 7 exhibits the correlation between objective (in the $\mathrm{x}$-axis) and subjective experiments (MOS in the y axis).

Table 1 summarizes the result of the correlation between the objectives (no reference metrics) and the subjective metrics MOS. From this table, we can note that the highest values of correlation coefficients for the suggested method followed by Entropy and the lowest values for correlation coefficients were for HDMHA algorithm. The objective score is fitted to the subjective score using the logistic fitting function:

$$
M O S_{f i t}=y_{f i t}=y o+\frac{a}{1+\left(\frac{x}{x o}\right)^{b}}
$$

The vector of parameters $n$ is given by:

$$
n=\left[\begin{array}{llll}
a & b & x o & y o
\end{array}\right]^{t}
$$

Where these parameters are find by minimizing the sum of squared differences between $M O S_{f i t}$ and subjective score $y$ by using:

$$
n=\min \sum\left(y_{f i t}-y\right)^{2}
$$

And same displays the scatter plots and fitted curves for the proposed and the other methods. The proposed achieve better fitting curve than other methods 
(Entropy, NIQE and TCE), in Fig. 7 because of the use of approximation, we notice that the scattered data approaches to linear with a positive slope, the better the scale This behavior was reflected in the suggested and TCE scales.

\section{Conclusion}

In this work, we present a novel and simple noreference IQA method for hazy distorting in the color images depending on the probability of the saturation, experiment results show the suggested method succeeded in measuring the no-reference quality of the hazy image with a high degree. The proposed algorithm is compared with four other no-reference methods, like Entropy, HDMHA, NIQE and TCE, by calculating the correlation coefficients (PCC, kendall and SCC) between the objective and subjective scores, as the proposed method had the highest correlation coefficients (0.8923 0.7170 and 0.8960$)$. This indicates that there is a good convergence between the proposed scale and the subjective quality assessment.

\section{Conflicts of Interest}

The authors declare no conflict of interest.

\section{Author Contributions}

Ahmed Rafid Hashim, Hazim G. Daway, and Hana H. kareem contributed to the design and implementation of the research, to the analysis of the results and to the writing of the manuscript.

\section{Acknowledgments}

We are grateful to the Department of Physics, College of Science, Mustansiriyah University for allowing us to use the system for capturing hazy images.

\section{References}

[1] W. Warner, R. Graham, R. Read, and S. Reutebuch, Small format aerial photography: Whittles Caithness, 1996.

[2] V. Kamble and K. Bhurchandi, "No-reference image quality assessment algorithms: A survey", Optik, Vol. 126, No. 11-12, pp. 1090-1097, 2015.

[3] Z. Wang, A. Bovik, H. Sheikh, and E. Simoncelli, "Image quality assessment: from error visibility to structural similarity", IEEE Transactions on Image Processing, Vol. 13, No. 4, pp. 600-612, 2004.
[4] Z. Wang, and A. C. Bovik, "A universal image quality index", IEEE Signal Processing Letters, Vol. 9, No. 3, pp. 81-84, 2002.

[5] Y. Lu, F. Xie, Y. Wu, Z. Jiang, and R. Meng, "No reference uneven illumination assessment for dermoscopy images", IEEE Signal Processing Letters, Vol. 22, No. 5, pp. 534-538, 2014.

[6] Z. Cao, Z. Wei, and G. Zhang, "A no-reference sharpness metric based on the notion of relative blur for Gaussian blurred image", Journal of Visual Communication and Image Representation, Vol. 25, No. 7, pp. 1763-1773, 2014.

[7] P. Marziliano, F. Dufaux, S. Winkler, and T. Ebrahimi, "Perceptual blur and ringing metrics: application to JPEG2000", Signal Processing: Image Communication, Vol. 19, No. 2, pp. 163 172, 2004.

[8] D. Li, T. Jiang, and M. Jiang, "Exploiting highlevel semantics for no-reference image quality assessment of realistic blur images", In: Proc. of the 25th ACM International Conf. on Multimedia, pp. 378-386, 2017.

[9] Z. Miao, F. Yuan, C. GAO, and E. Cheng, "New non-reference image quality evaluation method for underwater turbulence blurred images", In: Proc. of the Thirteenth ACM International Conf. on Underwater Networks \& Systems, pp. 1-8, 2018.

[10] S. Corchs, F. Gasparini, and R. Schettini, "No reference image quality classification for JPEGdistorted images", Digit. Signal Process, Vol. 30, pp. 86-100, 2014.

[11] R. Babu, S. Suresh, and A. Perkis, "No-reference JPEG-image quality assessment using GAPRBF”, Signal Processing, Vol. 87, No. 6, pp. 1493-1503, 2007.

[12] L. Liang, S. Wang, J. Chen, S. Ma, D. Zhao, and W. Gao, "No-reference perceptual image quality metric using gradient profiles for JPEG2000", Signal Processing: Image Communication, Vol. 25, No. 7, pp. 502-516, 2010.

[13] J. Zhang, S. Ong, and T. Le, "Kurtosis-based noreference quality assessment for JPEG2000 images", Signal Process.: Image Communication, Vol. 26, No. 1, pp. 13-23, 2011.

[14] M. Liu, G. Zhai, Z. Zhang, Y. Sun, K. Gu, and $\mathrm{X}$. Yang, "Blind image quality assessment for noise", In: Proc. of IEEE Int. Symp. Broadband Multimedia Syst. Broadcast (BMSB), pp. 1-5, 2014.

[15] E. Cohen and Y. Yitzhaky, "No-reference assessment of blur and noise impacts on image 
quality", Signal Image Video Process, Vol. 4, No. 3, pp. 289-302, 2010.

[16] Y. Liu, K. Yang, and H.Yan, "No-Reference Image Quality Assessment Method Based on Visual Parameters", Journal of Electronic Science and Technology, Vol. 17, No. 2, 2019.

[17] X. Chen, Q. Zhang, M. Lin, G. Yang, and C. He, "No-reference color image quality assessment: from entropy to perceptual quality", EURASIP Journal on Image and Video Processing, Vol. 2019, No. 1, p. 77, 2019.

[18] K. Gu, D. Tao, J. Qiao, and W. Lin, "Learning a no-reference quality assessment model of enhanced images with big data", IEEE Transactions on Neural Networks and Learning Systems, Vol. 29, No. 4, pp. 1301-1313, 2017.

[19] A. Moorthy and A. Bovik, "A two-step framework for constructing blind image quality indices", IEEE Signal Processing Letters, Vol. 17, No. 5, pp. 513-516, 2010.

[20] A. Mittal, R. Soundararajan, and A. Bovik, "Making a completely blind image quality analyzer", IEEE Signal Processing Letters, Vol. 20, No. 3, pp. 209-212, 2012.

[21] X. Pan, F. Xie, Z. Jiang, Z. Shi, and X. Luo, "No Reference Assessment on Haze for Remote Sensing Images", IEEE Geoscience and Remote Sensing Letters, Vol. 13, No. 12 , pp. 185 -1859, 2016.

[22] W. Shen, S. Hao, J. Qian, L. Li, "Blind Quality Assessment of Dehazed Images by Analyzing Information, Contrast, and Luminance", Journal of Network Intelligence, Vol. 2, No. 1, pp. 139146, 2017.

[23] H. Kareem, E. Daway, and H. Daway, "No Reference Quality Of Hazy Images Depending On Transmission Component Estimation", IIUM Engineering Journal, Vol. 20, No. 2, pp. 70-77, 2019.

[24] W. Zhang and X. Hou, "Incident Light Frequency-Based Image Defogging Algorithm", Mathematical Problems in Engineering, Vol. 2017, pp. 1-8, 2017.

[25] https://drive. google.com/open?id=1fDcZToFB5x71oSnvZDMPtpdFmWvArT5

[26] B. Series, "Methodology for the subjective assessment of the quality of television pictures", Recommendation ITU-R BT, pp. 500-13, 2012.

[27] S. Wine and R. Horne, The Color Image Processing Hand Book, International Thomson. 1998.

[28] A. I. McLeod, "Kendall rank correlation and Mann-Kendall trend test", $R$ Package Kendall, 2005 